\title{
Etude Des Déterminants De L'usage Des Services De L'administration Électronique Par Le Citoyen : Revue De Littérature
}

\author{
Satry Fadwa \\ Doctorante en Science de Gestion à la Faculté des Sciences Juridiques \\ Economiques et Sociales, \\ Ain Sebaâ, Université Hassan II-Casablanca, Morocco \\ Laboratoire de Recherche en Management, Information et Gouvernance \\ LARMIG \\ Belkadi Ez-zohra \\ Professeur en Sciences de Gestion à la Faculté des Sciences Juridiques \\ Economiques et Sociales, \\ Ain Sebaâ, Université Hassan II-Casablanca, Morocco \\ Laboratoire de Recherche en Management, Information et Gouvernance \\ LARMIG
}

Doi:10.19044/esj.2019.v15n4p284 ～URL:http://dx.doi.org/10.19044/esj.2019.v15n4p284

\section{Résumé}

L'administration électronique est définie comme étant la prestation des services publics aux citoyens à l'aide d'Internet et de technologie de l'information et de communication. La relation entre l'administration publique et le citoyen est dématérialisée au fil du temps afin d'offrir une prestation complète en matière de coûts, qualité et délais. L'objet de cet article est de présenter une revue de littérature, qui s'intéresse à l'étude des déterminants de l'usage des e-services publics par le citoyen, ceci à travers une démarche hypothético-déductive permettant d'élaborer un modèle de recherche qui sera testé dans des travaux ultérieurs dans le contexte Marocain. Ce sujet est abordé dans la perspective de répondre aux besoins du Citoyen d'un côté, et d'aider les décideurs publics à améliorer les services de l'administration électronique d'un autre côté.

Mots clés : E-administration, Citoyen usager, TIC, TAM, UTAUT 


\title{
Study of the Determinants of the Use of Electronic Administration Services by the Citizen: Literature Review
}

\author{
Satry Fadwa \\ Doctorante en Science de Gestion à la Faculté des Sciences Juridiques \\ Economiques et Sociales, \\ Ain Sebaâ, Université Hassan II-Casablanca, Morocco \\ Laboratoire de Recherche en Management, Information et Gouvernance \\ LARMIG \\ Belkadi Ez-zohra \\ Professeur en Sciences de Gestion à la Faculté des Sciences Juridiques \\ Economiques et Sociales, \\ Ain Sebaâ, Université Hassan II-Casablanca, Morocco \\ Laboratoire de Recherche en Management, Information et Gouvernance \\ LARMIG
}

\begin{abstract}
E-administration is defined as the delivery of public services to citizens through the use of the Internet, and information and communication technology. The relationship between the public administration and the citizen is dematerialized over time in order to provide a complete service in terms of costs, quality and deadlines. The purpose of this article is to present a literature review, which focuses on the study of the determinants of citizen use of public e-services, through a hypothetical-deductive approach to develop a research model that will be tested in later work in the Moroccan context. This topic is discussed with a view to meeting the needs of the Citizen on the one hand, and to helping policy makers to improve the services of electronic administration on the other hand.
\end{abstract}

Keywords: E-administration, Citizen User, TIC, TAM, UTAUT

\section{Introduction :}

L'administration électronique est désormais le pilier principal de la réforme de l'administration publique dans plusieurs pays. En effet l'administration en ligne et l'Internet ont engendré un changement radical au sein des organismes publics, dans le sens où la délivrance des services publics et le fonctionnement opérationnel de ces structures sont devenus associés à 
l'usage courant des e-services tout en bénéficiant du potentiel des TIC. Ce changement organisationnel se répercute non seulement sur le fournisseur des services publics mais bien évidemment sur le bénéficiaire de ces services qui est le citoyen usager. Ce dernier se trouve au fil du temps dans l'obligation d'utiliser les services publics électroniques.

En outre, l'administration électronique représente un avantage concurrentiel permettant l'amélioration des services publics par la garantie de la qualité dans la prestation, le raccourcissement des délais d'attente et de traitement, et la minimisation des coûts. Ainsi, elle contribue à la promotion de la transparence et la démocratie. De plus, elle respecte et préserve l'environnement par la réduction de l'utilisation des papiers, ce qui renforce son aspect sociétal. Cependant la mise en place de l'e-administration reste confrontée à de nombreux obstacles.

Dans cet article, nous allons présenter une revue de littérature sur les facteurs de l'usage des services de l'e-administration par le citoyen. Ce dernier est considéré dans cette donne du management public comme un client à satisfaire. Pour ce faire, nous allons dans un premier lieu définir le concept de l'administration électronique ainsi que son évolution. Nous allons aborder en deuxième lieu les modèles théoriques validés en matière d'acceptation d'utilisation des services en ligne. Ainsi, nous allons recourir à la théorie de l'action raisonnée (TRA) d'Ajzen et Fishbein (1980), la théorie du comportement planifié (TPN) (Ajzen, 1985), le modèle d'acceptation de la technologie (TAM) développé par Davis (1989) ainsi que l'extension du modèle d'acceptation de la technologie (TAM2) élaboré par Venkatesh et Davis (2000) et le modèle de diffusion de l'innovation (DOI) (Rogers, 2003). En outre, nous allons étudier la théorie unifiée de l'acceptation et de l'utilisation de la technologie (UTAUT) (Venkatesh et al. 2003). Finalement, nous allons exposer le modèle théorique basique de l'administration électronique en expliquant l'intérêt et la justification du choix de ce modèle de recherche.

\section{E-administration : Définitions et Concepts 1.1. Définitions et concepts :}

Le concept de l'administration électronique a vu le jour durant les années quatre-vingt-dix conjointement avec l'avènement d'internet (Grönlund 2002, 2005; Grönlund et Horan, 2005). En effet, elle désigne l'utilisation des TIC par l'administration publique. En outre, il existe plusieurs définitions pour la terminologie « administration électronique ». Cette dernière fait référence à la livraison d'informations et de services publics en ligne via Internet ou d'autres moyens numériques (West, 2000), et peut inclure des possibilités de participation politique en ligne (Mossberger, Tolbert et Stansbury, 2003). 
Pour sa part, Fang (2002) a défini l'administration électronique comme un moyen pour les autorités publiques d'utiliser les technologies de l'information et de la communication les plus innovantes, en particulier les applications Internet sur le Web, pour donner aux citoyens et aux entreprises un accès plus pratique aux informations et aux services publics, afin d'améliorer la qualité des services et offrir de plus grandes opportunités de participer aux institutions et aux processus démocratiques. De ce fait, l'administration électronique est prometteuse pour améliorer la prestation de nombreux types de services publics, tels que les transactions en ligne pour diffuser des informations sur le fonctionnement des organismes publics. Elle peut aussi améliorer la communication entre les citoyens et l'administration par courrier électronique, ce qui permet une participation plus directe à la prise de décision gouvernementale (Thomas et Streib 2003).

Par ailleurs, le rapport établi par l'OCDE en 2003, stipule que l'administration électronique peut être définie comme "l'utilisation des TIC, et en particulier d'Internet, comme outil pour parvenir à une meilleure gestion des affaires publiques". En outre, Moon et Norris (2005) fournissent une définition simple de l'administration électronique en la décrivant comme «un moyen pour fournir des informations et des services gouvernementaux». Pour Dada (2006), l'administration électronique traduit l'utilisation des technologies de l'information pour améliorer les relations entre l'administration publique et les citoyens dans différents domaines.

Ainsi, Coursey et Norris (2008) ont défini l'administration électronique comme l'utilisation de systèmes électroniques pour fournir des informations et des services publics à toutes les catégories de la société, 24 heures par jour et sept jours par semaine. L'organisation des Nations Unies (2010) définit l'administration électronique comme l'utilisation des TIC et son application par les établissements publics pour délivrer les informations et les services publics aux personnes. L'objectif de l'administration électronique est donc de fournir une gestion gouvernementale efficace de l'information au citoyen; une meilleure prestation des services aux citoyens et l'autonomisation des personnes par l'accès à l'information et la participation à la prise de décision en matière de politique publique. Les avantages qui en résultent, sont la lutte contre la corruption, une transparence accrue, une plus grande commodité, et une réduction des coûts (Banque Mondiale, 2011).

D'après ces définitions nous retenons que l'administration électronique est l'usage de l'internet et des technologies de l'information pour la prestation des services publics, et a pour vocation d'améliorer la qualité des services aux citoyens, garantir une gestion efficace de l'administration publique, instaurer une nouvelle gouvernance. 


\subsection{Evolution de l'administration électronique :}

De nombreux chercheurs ont distingué les étapes de l'évolution du eadministration (Hiller \& Bélanger, 2001; Layne \& Lee, 2001; UN \& ASPA, 2002), passant d'une phase d'information à une phase d'intégration totale. Ces étapes ont un aspect descriptif et chacune d'elles exige une certaine complexité au niveau des technologies de l'information et de communication, une priorité accordée au citoyen en tant que client à satisfaire et un changement organisationnel qui en découle (Holden, Norris, \& Fletcher, 2003; Moon, 2002).

Dans ce sillage, les étapes sont en nombre de quatre. Les trois premières: la phase d'information, la phase d'interaction et la phase de transaction, représentent des étapes de changement mineur. La quatrième d'intégration totale est une phase idéale de transformation d'où l'intégration totale des services intra-organisationnels et inter-organisationnels.

- La phase d'information ou de la présence initiale : c'est le premier pas vers le développement de chaque administration électronique. Pour Layne et Lee (2001), cette étape est formelle et elle nécessite l'utilisation d'Internet pour en bénéficier en termes de communication avec les parties prenantes. À ce stade, l'administration électronique offre des informations statiques sur ses différents départements et services qu'ils fournissent aux citoyens et aux entreprises, voire même la fourniture des informations plus spécialisées et dynamiques sous forme d'un catalogue (UN et ASPA, 2002). Cette phase marque une absence de communication bidirectionnelle avec chaque citoyen usager (St-Amant, 2005).

- La phase d'interaction : c'est une étape qui a intégré les limites de la phase initiale par sa concentration sur le développement d'une communication plus personnalisée avec les citoyens, avec la mise en place des prestations électroniques basées sur la messagerie électronique, les forums publics, les moteurs de recherche, les listes d'envoi...etc. À ce niveau l'interaction entre les citoyens et l'administration publique désormais importante (Hiller et Bélanger, 2001; UN et ASPA, 2002).

Grâce à cette émergence vers une communication bidirectionnelle accentuée par des prestations personnalisées, les administrés ; des particuliers ou des entreprises ; peuvent accéder à l'information selon leurs besoins et leurs intérêts. Afin de garantir plus de sécurité et de confidentialité, des mots de passe sont utilisés pour avoir accès à certains services. C'est une étape qui renforce la transparence de l'État et stimule la confiance et la participation citoyenne dans la gestion de la chose publique. Pourtant, elle est caractérisée par un manque de mise en œuvre des démarches de traitement des transactions 
en ligne ce qui ne permet pas de diminuer les délais de traitement complet d'un service.

- La phase de transaction : est une continuité de la deuxième étape, elle s'intéresse à la création d'un portail global et unique des services publics répondant à un besoin spécifique et en se basant sur les attentes et les exigences de l'usager pour faire la conception du portail. En introduisant des télés-procédures de paiement électronique sécurisé, tout en facilitant les transactions dont le paiement des impôts et taxes (Hiller et Bélanger, 2001; Layne et Lee, 2001).C'est la voie vers la simplification et l'automatisation des procédures qui apparaît dans la gestion électronique totale des transactions. Commençant par la collecte, le traitement des données, en arrivant à la validation et la conservation des données. Parmi les avantages de cette phase; le passage remarquable d'une relation de guichet vers une relation de réseau limitant l'utilisation de la paperasse, et bénéficiant des délais d'attente trop courts et des services complets disponibles immédiatement.

- La phase d'intégration : certains auteurs décomposent cette phase en intégration verticale et horizontale (Layne et Lee ,2001). Cependant St-Amant (2005), distingue entre une intégration intraorganisationnelle et inter-organisationnelle. De leur part, Hiller et Bélanger (2001), Un et Aspa (2002) avancent que cette phase préconise une intégration totale des services publics. En effet, un seul portail fera l'objet d'une réponse aux différents besoins des citoyens concernant divers services publics, dans la mesure où il aura l'accès à une multitude de services à travers la consultation d'une page Web unique à l'aide d'un identifiant et un mot de passe. Le règlement de ces services peut être effectué dans une seule facture regroupée. Gupta et al (2003) expliquent que les systèmes sont intégrés à différentes fonctions « Real one stop shopping », ce qui légitime une intégration motivée par une vision d'efficacité et d'efficience dans l'usage des TIC.

Nous assistons donc à une intégration totale en termes de prestations électroniques des services publics dans une plateforme unifiée. Ceci va permettre au citoyen de bénéficier d'une prise en charge globale de la demande d'un service même si cette dernière impose l'intervention de plusieurs organismes. L'usager bénéficiera d'une manière personnalisée d'un accès sécurisé aux services sans prendre en considération le temps et l'espace.

La fourniture électronique des services publics évolue significativement d'un portail d'informations à un portail totalement intégré. Par ailleurs, cette évolution nécessite une intégration intra-organisationnelle et interorganisationnelle au niveau des processus, tout en mettant le citoyen au cœur 
de cette transformation de l'administration publique. D'où St-Amant (2005) précise l'importance d'opérer selon une approche orientée vers le citoyen comme étant un client à satisfaire en termes de qualité, délais et coûts de délivrance de services, et complémentarité des services.

En outre cette évolution, introduit à chaque phase deux dynamiques primordiales qui apparaissent dans la sophistication technologique et le changement organisationnel. Finalement pour layne et lee (2001), ces étapes constituent le bon chemin à suivre pour promouvoir une initiative complètement intégrée d'administration électronique, qui facilite le «mode d'achat unique » pour le citoyen.

\section{Revue de littérature :}

\subsection{La Théorie de l'action raisonnée (TRA) :}

La théorie de l'action Raisonnée (TRA) a été développée par Ajzen et Fishbein (1980) dans un cadre de psychologie sociale. Elle représente le premier modèle utilisé pour expliquer l'acceptation de la technologie. Ces deux théoriciens ont visé à développer une théorie qui pourrait prédire, expliquer et influencer le comportement humain, tout en se concentrant sur la relation entre plusieurs variables qui forment un modèle de prédiction d'intentions et de comportements spécifiques, telles que : les croyances, les attitudes, les normes subjectives, l'intention et les comportements. Ainsi, Ajzen et Fishbein (1980) mentionnent l'importance du TRA en tant que fondement théorique, il est le modèle approprié pour étudier et expliquer les déterminants du comportement de l'utilisateur dans une grande variété de domaines.

La TRA, stipule que le déterminant principal du comportement n'est pas l'attitude de la personne envers le comportement, mais son intention d'exécuter le comportement. Cette intention comportementale est déterminée par deux facteurs :

$\checkmark$ Le premier facteur est l'attitude de la personne envers le comportement, qui est la mesure dans laquelle la personne a une évaluation positive ou négative du comportement.

$\checkmark$ Le deuxième facteur est la norme subjective, c'est-à-dire la pression sociale perçue pour exécuter ou ne pas exécuter le comportement.

Ces deux facteurs sont soutenus par des ensembles de croyances. En effet, pour l'attitude, les croyances sont des croyances comportementales qui concernent la probabilité perçue que l'exécution du comportement permet la réalisation et l'évaluation de certains résultats. En ce qui concerne la norme subjective, les croyances sont des croyances normatives axées sur la pression sociale perçue de certains référents et la motivation de la personne à se conformer à ces référents. Selon Ajzen et Fishbein (1980), l'intention 
comportementale (BI) est le principal prédicteur du comportement que l'attitude.

Or, Ajzen (1985) a signalé certaines limites dont la correspondance. Ainsi, Sheppard, Hartwick et Warshaw (1988), indiquent que l'attitude et l'intention doivent s'accorder sur l'action, la cible, le contexte, le cadre temporel et la spécificité, dans la mesure où la théorie prédit un comportement spécifique. En outre la principale limite de cette théorie réside dans l'hypothèse que le comportement est sous contrôle volontaire. Autrement dit, les décisions irrationnelles, les actions habituelles ou tout comportement qui n'est pas consciemment pris en compte ne peuvent pas être expliqués par cette théorie, puisque cette dernière ne s'applique qu'à un comportement consciemment pensé à l'avance.

\subsection{La Théorie du comportement planifié (TPB) :}

Après avoir constaté certaines limites dans la théorie de l'action raisonnée (TRA), Ajzen (1985) a résolu le problème du contrôle volontaire, à travers la proposition d'une extension de la TRA, nommée la théorie du comportement planifié (TPB). Selon Ajzen (1991) la TPB est un modèle qui est largement utilisé pour prédire et expliquer le comportement humain avec la prise en considération des rôles des membres organisationnels, individuels et des systèmes sociaux dans ce processus. De ce fait la TPB a été conçue pour prédire des comportements qui ne sont pas entièrement sous contrôle volontaire en incluant des mesures de contrôle comportemental perçu (Ajzen, 1985, 1991). En outre, la TPB se distingue de la TRA par son inclusion de l'élément du contrôle comportemental perçu (PBC) qui tient compte des situations où un individu a un contrôle incomplet sur le comportement. Cela diffère selon les situations et les actions (Ajzen, 1991). Ainsi, La TPB met la composante de PBC dans un champ global de relations entre : croyances, attitudes, intentions et comportements. Davantage le PBC est censé influencer à la fois l'intention et le comportement. En effet, L'effet du PBC sur le comportement peut être direct ou interactif c'est-à-dire par le biais de l'intention comportementale. Par conséquent, les intentions seules devraient suffire à prédire le comportement, quand la situation ou le comportement donne à la personne la possibilité de contrôler complètement la performance comportementale, c'est ce qui a été évoqué dans la TRA. Également, Ajzen (1991) avance que quand l'intention comportementale (BI) seule ne représenterait qu'une petite partie de la variance dans le comportement dans certaines conditions, le PBC devrait être indépendamment prédictif du comportement.

D'ailleurs, les intentions et le PBC sont nécessaires pour prédire le comportement. Pourtant, l'un peut être plus important que l'autre selon la prédominance de certaines conditions. La TPB traite des antécédents de 
l'attitude, des normes subjectives et du contrôle comportemental perçu, en vue d'expliquer et de prédire le comportement. Ainsi, elle postule que le comportement est une fonction des croyances saillantes pertinentes à ce comportement. Ces croyances saillantes sont considérées comme les déterminants prédominants des intentions et des actions d'une personne.

Malgré les apports de la TBP, elle a été critiquée également par Taylor et Todd (1995), par le fait d'utiliser une variable (PBC) comme agent préventif pour tous les éléments non contrôlables du comportement. De ce fait, les croyances derrière le PBC ont été agrégées pour créer une mesure pour cela, cette agrégation a été critiquée par l'absence d'identification des facteurs spécifiques permettant la prédiction du comportement. De leur part, Armitage et Conner ( 2001), stipulent que le manque des variables démographiques dans un modèle psychologique basé sur un processus interne, et la considération que toutes les personnes ont une connaissance sur le modèle , ne déterminent pas le changement du comportement. De plus, la TBP n'explique pas toutes les variables ce qui a appuyé la critique que ce modèle ne cherche pas la relation d'intention et du comportement.

\subsection{Le modèle d'acceptation de la technologie (TAM) :}

Parmi les modèles les plus connus qui permettent de fournir une explication du comportement des individus envers l'adoption et l'utilisation des TIC, nous citons le modèle d'acceptation de la technologie (TAM : Technology Acceptance Model). Ce modèle est développé par Davis (1989), il représente une révision et une adaptation des théories précédentes, et il a pour utilité d'expliquer les déterminants d'acceptation et d'utilisation de la technologie par l'usager, c'est-à-dire les raisons qui le poussent à faire appel aux TIC. De plus, il vise à préciser les facteurs clés d'influence qui contribuent à cette adoption.

Le TAM est axé sur deux facteurs :

$\checkmark \quad$ L'utilité perçue (PU): selon Davis (1989), cette utilité signifie le degré auquel une personne croit que l'utilisation des TIC et des SI, permettra d'augmenter son rendement professionnel, ainsi que sa productivité avec efficacité et efficience.

$\checkmark$ La facilité d'utilisation perçue (PEOU): chez Davis (1989), ce concept indique le degré auquel une personne pense que l'utilisation des TIC ne nécessite pas d'efforts.

Davis, Bagozzi, et Warshaw (1992), déclarent que le TAM révèle les antécédents de l'utilisation des TIC à travers des croyances. Ceci, a renforcé la puissance et l'importance du TAM dans ce domaine. En effet, l'utilisation des TIC par l'usager est influencée directement par son intention comportementale, qui est à sa tour déterminée à la fois par l'utilité perçue de l'individu et son attitude envers l'usage des TIC. Autrement dit, le TAM 
explique l'usage effectif des TIC par l'intention, qui est influencée également par l'utilité et l'attitude, c'est-à-dire, la personne pense que son rendement au travail est en amélioration quelle que soit la nature de ses sentiments positifs ou négatifs (Davis et al. 1992).

En outre, ce modèle contient des variables externes précisées par Davis et Venkatesh (1996), d'où nous trouvons les caractéristiques objectives de la conception du système, la formation, l'auto-efficacité informatique, la participation des utilisateurs à la conception et à la mise en place des projets basés sur les TIC. Ces variables ne se dotent pas d'un caractère exhaustif, dans la mesure où l'évolution de ce modèle est perpétuelle au fil des années, traduite par l'introduction de nouvelles variables: la qualité du système, la compatibilité, l'anxiété informatique, le plaisir, le soutien informatique, et l'expérience d'utilisation (Lee, Kozar et Larsen, 2003).

Comme tout modèle, le TAM a été critiqué par de nombreux théoriciens. Venkatesh (2000) avance que ce dernier ne permet ni l'explication de l'acceptation des TIC par les individus, ni la détermination des critères qui ont un effet sur la facilité d'utilisation. Le TAM ne donne que des conseils minimes relatifs à la manière d'influencer l'utilisation à l'aide de la conception et de la mise en œuvre, ce qui engendre un manque d'orientation et par la suite le ralentissement du développement en la matière. De leur part, Legris et al, (2003) abordent deux limites ; la première désigne l'hypothèse qui stipule que l'usage réel est reflété par l'usage autodéclaré, et la deuxième s'intéresse à la pauvreté de la cible d'où l'étude ne concerne qu'une catégorie spécifique ce qui ne permet pas une généralisation des résultats. Davantage, les incohérences entre les travaux antérieurs et le pouvoir explicatif du modèle, étaient les principaux critiques de Sun et Zhang (2006).

\subsection{Le TAM 2 : le modèle d'acceptation de la technologie révisé}

Pour surmonter les lacunes de TAM 1, Venkatesh et Davis (2000), ont essayé d'apporter des modifications sur le modèle d'origine, pour qu'il soit TAM 2 comme étant son extension. Ce modèle s'est avéré un cadre efficace pour prédire l'utilisation des TIC. En outre, le TAM 2 inclue deux déterminants supplémentaires, nous distinguons entre les processus d'influence sociale et les processus cognitifs instrumentaux.

Concernant les premiers processus, il s'agit des processus émotionnels qui contiennent: les normes subjectives, l'aspect volontaire en termes d'usage des TIC, l'image sociale de l'individu et l'expérience. Cependant, ce dernier élément d'expérience d'utilisation ne fait pas partie réellement du processus d'influence sociale mais il est juste relié à ce système, de plus elle remplacera les normes subjectives (Davis et Venkatesh, 2000).

Le deuxième déterminant représenté par les processus cognitifs instrumentaux couvre: la pertinence au travail, en d'autre termes 
l'appréciation et la perception de l'individu sur les apports et les bénéfices de l'adoption des TIC. En ajoutant aussi, la qualité des résultats introduits de cet usage, la démontrabilité de ces résultats, et la facilité d'utilisation perçue.

Venkatesh et Davis (2000), ont confirmé que les influences sociales et les processus instrumentaux cognitifs ont un effet important sur l'utilisation et l'acceptation des TIC par les usagers potentiels, soit dans un cadre volontaire ou contraint.

\subsection{Le TAM 3 : Extension du TAM 1 et du TAM 2 :}

Le TAM 2 s'intéressait seulement aux déterminants associés à l'utilité perçue et à l'intention d'usage. La raison pour laquelle, Venkatesh (2008) a poursuivi ses recherches et a collaboré avec Balla (2008) tout en reprenant les anciennes versions du TAM en vue de revoir le modèle. Cela, a contribué à l'apparition de TAM 3 comme fruit de collaboration (Venkatesh et Balla, 2008). Ce dernier a été testé dans des environnements réels d'implémentation technologique. En effet, cette adaptation a été couronnée par l'ajout des déterminants qui se concentraient beaucoup plus sur la facilité d'utilisation perçue, d'où Venkatesh a distingué entre deux types de variables : variables d'ancrage et ceux d'ajustement.

Pour Venkatesh et Balla (2008), les individus sont influencés par l'autoefficacité qui renvoie à leurs croyances en matière de capacité d'utiliser ou ne pas utiliser les TIC, l'anxiété vis-à-vis de l'usage des TIC, la perception d'un contrôle externe et l'appréciation de l'usage des TIC (amusement, plaisir). Tous ces éléments constituent un panier de variables d'ancrage permettant de saisir et comprendre la facilité d'utilisation perçue, d'une part. D'autre part, l'ajustement est introduit par le niveau d'utilisation des TIC par l'individu afin de réaliser des objectifs bien déterminés avec efficacité et efficience, c'est ce qu'on appelle l'utilisabilité ainsi que le caractère ludique de cet usage.

\subsection{Le modèle motivationnel :}

Davis, Bagozzi et Warshaw (1992), ont élaboré un modèle motivationnel qui sert à saisir et à expliquer l'adoption et l'usage des nouvelles technologies à travers la motivation interne et celle externe:

$\checkmark$ La motivation intrinsèque désigne la perception selon laquelle l'usager ne voudrait effectuer une activité que pour le processus d'exécution en lui-même (Kéfi, 2010).

$\checkmark$ La motivation extrinsèque renvoie à la perception selon laquelle l'individu n'a volonté de faire une activité que si elle représente un outil de réalisation des gains et des profits, par exemple: la rémunération, la reconnaissance, l'estime, et l'amélioration de la performance professionnelle. 


\subsection{La théorie sociale cognitive (TSC)}

Durant les années 80, Bandura a concentré ses recherches sur le domaine de l'apprentissage social. En outre, ces travaux ont été développés pour introduire un nouveau concept celui du sentiment d'efficacité personnelle (Bandura, 1986,1997). Ainsi, l'affinement et les apports de Bandura ont évolué avec le temps, ce qui a conduit vers une intégration des concepts fondamentaux dans une théorie qui répond à des problématiques liées à la psychologie sociale. C'est la théorie sociale cognitive. Cette théorie est déterminée par une combinaison de facteurs : cognitifs, environnementaux et comportementaux. Elle a pour utilité d'expliquer le comportement de l'individu face à l'usage de la technologie.

Bandura (1977, 1986), insiste sur la notion d'interaction entre les trois facteurs mentionnés ci-dessus, sous l'hypothèse que le comportement n'est pas déterminé seulement par l'influence d'autrui et de son entourage, mais il est issu également des relations réciproques entre les trois éléments (facteurs cognitifs, environnementaux et de comportement) dont l'interaction joue le rôle de déterminisme. En effet, l'apprentissage social sera pénible et incertain si les gens comptent uniquement sur leurs propres pratiques pour orienter l'exécution de leurs tâches. Ce chercheur, a déclaré davantage que le comportement humain est appréhendé par un modelage, via l'observation des autres, la formation des idées sur la réalisation de nouveaux comportements, ainsi que le codage de cette information constitue une orientation vers l'action.

L'apport de la théorie de Bandura a été reconnu par la concentration sur le concept du SEP : Sentiment d'efficacité Personnelle. Ce terme se réfère à l'ensemble des jugements faits par les individus sur leur capacité de réaliser et d'effectuer des actions nécessaires à l'atteinte des performances attendues (Bandura, 1986), d'où le comportement est régi par les croyances personnelles vis-à-vis de la capacité à mener des actions réussies, et par les croyances relatives à la capacité d'employer des éléments cognitifs, la motivation et les comportements requis afin de faire un contrôle sur les faits vécus (Bandura, 1986 ; Wood et Bandura, 1989). De ce fait, le SEP est la boussole qui guide le choix des actions et de l'environnement des individus, de plus, il détermine l'effort exigé, la persistance, les feed-back émotionnels envers les problèmes, ainsi que les pensées favorables et défavorables.

Quant à la distinction entre les individus qui ont un faible SEP et ceux avec un niveau élevé du SEP. Bandura (1995), montre que les premiers sont des personnes qui ne résistent pas à l'échec, ne prennent pas des risques, ils renoncent à faire des missions perçues difficiles, ils se qualifient par une faible implication à la réalisation des objectifs escomptés, et ils ont un manque de confiance en leur capacité ce qui nuit à l'achèvement des résultats attendus d'une manière performante et détruit leur bien-être psychique ( stress..). Cependant, les personnes ayant un fort SEP, elles confrontent les problèmes, 
elles apprennent de l'échec, elles sont dotées de l'estime de soi et une grande confiance à leur capacité, ce qui leur permet de relever les défis et réussir leurs objectifs tout en bénéficiant des expériences potentielles et maitrisées. Également, le déploiement d'effort. Ceci, renvoie à l'auto-efficacité qui stimule l'accomplissement personnel et assure le bien-être social.

En outre, Bandura (1997), montre que le SEP mène vers la persévérance plutôt que les intérêts, ce qui appuie l'asymétrie entre les attentes de l'individu et son SEP; plus que le degré de réussite en matière de la réalisation d'une tâche dans un champ bien déterminé, est élevé plus que la préoccupation autour de ce champ est renforcée. En résumé, Bandura met le point sur l'apprentissage par l'observation et le modelage de l'autre. Ce qui montre que l'individu apprend à travers l'observation, l'attitude, la réaction émotionnelle et les comportements résultants des autres. Cette théorie vise à expliquer différents types de comportement, cette prédiction exige un modelage efficace axé sur un ensemble de construits (Bandura, 1988):

\begin{tabular}{|l|l|}
\hline \multicolumn{1}{|c|}{ Elément } & \multicolumn{1}{|c|}{ Signification } \\
\hline L'attention & $\begin{array}{l}\text { L'apprenant doit porter beaucoup d'attention aux } \\
\text { caractéristiques des comportements de modèle, afin } \\
\text { d'apprendre de nouvelles choses. Ainsi, l'attention est } \\
\text { influencée par : la complexité, la prévalence, la valence de } \\
\text { l'affect, les caractéristiques de l'apprenant et la valeur } \\
\text { fonctionnelle }\end{array}$ \\
\hline La rétention & $\begin{array}{l}\text { Elle nécessite une bonne mémoire, dans la mesure où } \\
\text { l'apprenant doit se rappeler des détails du comportement, } \\
\text { les images mentales, et le codage symbolique. }\end{array}$ \\
\hline La reproduction & $\begin{array}{l}\text { La stimulation de l'observation des comportements des } \\
\text { autres par l'apprenant permet de reproduire une } \\
\text { performance significative. En effet, la continuité de la } \\
\text { pratique améliore les capacités de l'apprenant. }\end{array}$ \\
\hline La motivation & $\begin{array}{l}\text { La réussite de l'apprentissage est associée à la motivation } \\
\text { de l'individu en matière de reproduction du comportement }\end{array}$ \\
\hline
\end{tabular}

Le modèle de Bandura a posé plusieurs concepts majeurs dont le sentiment d'efficacité personnelle, qui stipule que le comportement humain est influencé par son environnement, ses relations sociales et les comportements des autres. Ainsi, il représente un déterminant qui pousse la personne à la prise de décision, à l'action et à la réussite.

\subsection{La théorie de la diffusion de l'innovation (DOI) :}

Cette théorie, trouve ses origines dans les travaux de Rogers (1995). Elle vise à expliquer la diffusion des innovations au sein de la société et l'adoption de ces innovations par les individus. Cette adoption et diffusion sont expliquées par des facteurs exogènes et endogènes. Les premiers résident dans le cadre d'insertion de l'innovation telle que le nombre d'utilisateurs des produits innovants, et la disponibilité de ces innovations. Les seconds facteurs 
sont qualifiés d'endogène qui influencent la rapidité de la diffusion ou du rejet de l'innovation (Rogers, 2003; Karâa et Morana, 2011) :

$\checkmark$ L'avantage relatif: le degré auquel l'individu perçoit l'innovation comme source de bénéfice et des apports avantageux, qui peuvent être mesurés selon une approche économique ou selon un aspect de prestige social et satisfaction.

$\checkmark$ La compatibilité: Elle désigne que l'innovation doit être adéquate avec les valeurs, les pratiques sociales, les expériences et les attentes des usagers. En d'autres termes, plus l'innovation est compatible avec ces normes plus son adoption sera facile et rapide, et dans le cas contraire l'adoption connaîtra des entraves et nécessitera plus de temps pour l'adopter.

$\checkmark$ La complexité: le degré de difficulté perçu par les individus pour assimiler et utiliser l'innovation, plus la personne croit que cette innovation est difficile plus il renonce à son adoption et inversement.

$\checkmark$ La testabilité: qui renvoie à la possibilité d'essai et de l'expérimentation de l'innovation avant de procéder à son utilisation effective, ce qui permet d'éviter les risques liés au dysfonctionnement ou de maintenance.

$\checkmark$ La visibilité : qui signifie l'observation des résultats issus de l'innovation, plus ces résultats sont visibles plus l'usage de l'innovation est flexible.

Il est important ici, d'aborder la différence effectuée par Rogers (2003) entre la diffusion et l'adoption de l'innovation. La première est liée à la société c'est-à-dire au groupe, d'où la définition est la suivante: « la diffusion est le processus par lequel une innovation se transmet par le biais des canaux au fil du temps entre les membres d'un système social ». Alors que 1'adoption est associée à l'individu, et elle est définie comme la décision de faire pleinement d'usage et tirer profit d'une innovation, tout en la considérant comme une meilleure ligne de conduite disponible.

Or, la théorie de diffusion de l'innovation a connu quelques limites, qui figurent dans les critiques d'Attewell (1992) et Clarke (1999). Pour le premier chercheur, cette théorie donne beaucoup d'importance à la demande de l'innovation qu'à l'offre de cette dernière, ainsi, la demande est déterminée par la connaissance du potentiel de l'innovation et son adoption. Il stipule que les stratégies marketing et d'éducation adoptées par les organisations permettent d'influencer la diffusion de l'innovation, ce qui rend le caractère limitatif de cette diffusion parce que certaines entreprises connaissent une absence de telles stratégies. En outre, la complexité d'une innovation constitue une barrière devant son utilisation par l'usager même s'il connaît ses avantages (Attewell, 1992). Clarke (1999) a formulé des critiques à propos de la faiblesse de cette théorie en matière de pertinence des résultats en expliquant 
que cette théorie donne lieu à des hypothèses rejetées facilement et elle reste toujours associée à la culture de son champ d'application. En effet, c'est une théorie impuissante pour prédire et élaborer des recommandations pour accroitre et accélérer l'adoption (Clarke, 1999).

\subsection{La théorie d'influence sociale, des comportements interpersonnels et modèles d'utilisation des PC :}

Nous avons intégré ces deux théories parce qu'elles ont la même préoccupation et elles se focalisent toutes les deux sur des facteurs sociaux, pour expliquer le comportement des individus à l'égard des TIC et de l'usage des services électroniques. D'une part, la théorie d'influence sociale est développée par Schmitz et Fulk (1991), elle a pour utilité de montrer que l'environnement social influence le comportement de la personne en matière d'acceptation et d'adoption de la technologie, et elle trouve ses fondements dans la théorie sociale cognitive. En effet, ces deux théoriciens ont été inspirés de Bandura (1977), et ils ont mis le point sur deux facteurs sociaux. Le premier est l'influence sociale qui indique la perception de l'individu sur la technologie qui résulte de l'impact des règles sociales et des attitudes des collaborateurs au travail. Ainsi, le deuxième s'intéresse aux expériences vécues dans le passé en utilisant les SI par l'usager potentiel.

D'une autre part, Triandis (1971) a réalisé un exploit en s'appuyant sur des facteurs ayant un caractère social, attitudinal et affectif pour appréhender le comportement humain envers l'utilisation des TIC. Ses travaux ont été reconnus par la théorie des comportements interpersonnels. De ce fait, ces facteurs comprennent:

$\checkmark$ Les facteurs sociaux: 1'être humain est sociable par nature, il vit au sein d'une communauté, il a des attentes et des besoins, il a aussi des points de vue et des attitudes. Donc il agit et interagit selon ce champ d'appartenance et selon la pensée de ses parties prenantes.

$\checkmark$ Les conditions facilitatrices: des éléments qui assurent la facilité du comportement concerné. Elles regroupent la qualification du personnel de l'administration publique en matière des SI et la disponibilité des infrastructures technologiques.

$\checkmark$ L'attitude: renvoie aux croyances et aux ambitions et attentes des usagers envers les TIC.

$\checkmark \quad$ L'affect: reflète un ensemble de sentiments que relie la personne à un comportement bien déterminé. Ces sentiments peuvent exprimer une joie, un dégout, un plaisir, une gaité, un mécontentement, ou une haine.

$\checkmark$ Les habitudes: Elles ont une influence sur l'intention et l'attitude de la personne. En outre, elles représentent les actions répétitives et automatiques exercées par l'individu, et elles naissent d'un 
apprentissage comme elles peuvent avoir lieu sans instruction préalable.

$\checkmark$ Les conséquences perçues : sont des résultats évalués et valorisés par l'individu suite aux conséquences incertaines de son comportement.

Il s'agit ici de bien prendre en considération les théories à base sociale pour prédire et saisir les facteurs sociaux déterminant l'acceptation et l'utilisation des e-services publics par les citoyens. Au fait, il y a quelques chercheurs qui ont creusé et se sont inspirés de Triandis (1971) pour élaborer le modèle de l'utilisation des PC (Thomson et al, 1994). C'est une mise en place de la théorie interpersonnelle dans un contexte informatique pour expliquer l'usage des PC par le personnel qualifié. Ce modèle s'est attaqué non seulement aux facteurs sociaux, conditions facilitatrices, et affect, mais également à la complexité qui désigne le niveau de difficulté perçue par l'individu en termes d'assimilation et d'utilisation des SI, et à la compatibilité de la tâche qui signifie le degré selon lequel la personne pense que l'utilisation de SI augmente son rendement professionnel.

\subsection{La théorie unifiée de l'acceptation de la technologie UTAUT:}

Comme son nom l'indique, la théorie unifiée de l'acceptation de la technologie développée par Venkatesh, Morris, et Davis (2003), représente une combinaison de huit modèles précédents avec des affinements et modifications. Ces modèles sont le TRA, le TPB, le TAM, le TAM2, le modèle de motivation (MM), le modèle d'utilisation du PC (MPCU), le DOI et la théorie cognitive sociale (SCT). L'UTAUT constitue un modèle courant qui a pour objet d'expliquer les intentions des utilisateurs des nouvelles technologies et l'usage réel de ces dernières.

À travers les modèles individuels évoqués précédemment, qui visent à prédire l'adoption des TIC par l'individu, ainsi que les facteurs qui influent sur cet usage. Venkatesh et al. (2003) ont fondé ce modèle consolidé et synthétique pour donner une image claire et globale du processus d'acceptation. En effet, l'explication du comportement de l'usager nécessite l'intervention d'une multitude de variables. La théorie a introduit quatre concepts fondamentaux : la performance attendue, l'effort attendu, l'influence sociale et les conditions facilitatrices.

De ce fait, la performance attendue, l'effort attendu, l'influence sociale sont des déterminants directs de l'intention qui explique par la suite l'usage effectif de l'individu des TIC. Les conditions facilitatrices influent, quant à elles, directement sur le comportement d'utilisation sans passer par l'intention (Venkatesh et al. 2003). De plus, la théorie unifiée comprend également des variables modératrices telles que le sexe, l'âge, l'expérience et le volontarisme (critère d'usage volontaire ou obligatoire). Par ailleurs, l'UTAUT explique 
l'influence des caractères personnels et sociodémographiques sur l'utilisation de la technologie. À cet effet, plusieurs recherches ont accentué les travaux de Venkatesh et al (2003) en assurant que la relation entre l'utilité perçue, la facilité d'utilisation et l'intention d'utiliser peut-être modérée par l'âge, le sexe et l'expérience. Dans la mesure où les interactions entre l'utilité perçue et l'intention d'utiliser varient avec l'âge et le sexe, la technologie est désormais plus importante chez les hommes et les jeunes travailleurs d'un côté. D'un autre côté, Venkatesh et al (2003) ont montré que l'effet de la facilité d'utilisation perçue sur l'intention est modéré par le sexe et l'âge, ce qui a permet de constater que cette influence et omniprésente chez les femmes et les travailleurs âgés, pourtant elle connaît une diminution avec l'expérience.

Les construits de la théorie unifié de l'acceptation de la technologie de Venkatesh et al (2003), sont définis comme suit :

$\checkmark \quad$ La performance attendue (PE): est le degré auquel l'individu croit que l'utilisation d'un système (TIC) peut l'aider à augmenter ses bénéfices et améliorer sa performance professionnelle

$\checkmark \quad$ L'effort attendu (EE): est le degré de facilité relative à l'utilisation du système(TIC)

$\checkmark$ Influence sociale (IS): l'impact des normes et des attitudes de l'entourage de l'utilisateur sur sa perception envers l'usage des SI

$\checkmark$ Les conditions facilitatrices (CF): est le niveau auquel la personne pense que son utilisation des SI est soutenue par la présence et la disponibilité d'une infrastructure technique et organisationnelle importante.

Nous remarquons que la performance attendue (PE) figurant dans le modèle UTAUT est le résultat d'un dosage entre cinq variables : l'utilité perçue, la motivation extrinsèque, l'ajustement au travail, l'avantage relatif et les résultats attendus. Ainsi, la validation de modèle de Venkatesh et al. (2003) est renforcée par ce prédicteur de la performance attendue vue sa signification et efficacité dans un cadre contraint ou volontaire. En outre, l'effort attendu (EE) est issu de la facilité d'utilisation perçue et de complexité. Il y a lieu de rappeler que la facilité d'utilisation perçue figure dans le modèle de Davis (1989) influence l'acceptation de la technologie et l'utilité perçue. Davantage, l'effort attendu a contribué à la validation de l'UTAUT à travers sa signification dans les deux types de cadre d'utilisation obligatoire et volontaire. Par conséquent l'utilisation courante des TIC garantit une aisance et une satisfaction en la matière. Quant à L'influence sociale, elle englobe l'impact des opinions des autres sur la perception d'usage de l'individu, la culture subjective, les rapports interpersonnels ainsi que les interactions avec son entourage d'appartenance. De plus, le degré de l'utilisation perçue d'une innovation pour améliorer son image ou son statut dans son système social (Venkatesh et al. 2003). Nous constatons, que ce construit fait références aux 
travaux déjà cités qui ont mis en avant la norme subjective, les facteurs sociaux et l'image. De ce fait, Venkatesh et al. (2003) ont conclu que l'influence sociale est seulement significative dans le contexte d'adoption obligatoire et ce n'est pas le cas dans le contexte volontaire. Tandis que les conditions facilitatrices (FC) sont représentées par le contrôle comportemental perçu, les conditions facilitatrices et la compatibilité avec les modèles antérieurs. De la même manière, Venkatesh et al (2003) déclarent que les conditions facilitatrices sont à leur tour significatives dans l'environnement contraint et volontaire d'utilisation durant la durée d'usage, cependant, son influence sur l'intention est absente après cette période d'utilisation.

Finalement, la mesure apportée par l'un des modèles originaux en matière d'acceptation de la technologie a représenté au maximum $40 \%$ de la variance d'intention d'utilisation, ce qui a stimulé la puissance du modèle de l'UTAUT qui a été capable de représenter $70 \%$ de la variance de l'intention d'utilisation. Par conséquent, la limitation de la validité du contenu révélé par les théoriciens est due aux procédures de mesure, et recommandent une validation complète du contenu et un développement approprié de cette validation par les travaux ultérieurs (Venkatesh et al, 2003). Ce dernier constat, a donné naissance à une extension de l'UTAUT traduite par l'UTAUT 2 développée par Venkatesh, Thong et $\mathrm{Xu}$ (2012). Ils ont repris la version originale de l'UTAUT (Venkatesh et al, 2003), et ils ont ajouté des facteurs émotionnels tels que le plaisir et l'hédonisme, en incluant aussi le coût financier et les habitudes qui apparaissent dans les schèmes, les routines et les automatismes.

\section{Le Modèle de Recherche :}

L'administration était depuis toujours le centre de critiques de toute la société civile, et plus particulièrement le citoyen usager de ses services publics. Dans ce sillage, l'avènement d'Internet et le développement des TIC ont constitué une opportunité à saisir afin de remédier aux dysfonctionnements de l'administration publique. En effet, l'administration électronique marque un passage très important d'une relation de guichet à une relation de réseau entre le citoyen et l'administration publique, en vue de lui assurer une prestation de meilleure qualité et de lui garantir une satisfaction totale.

En outre, l'administration électronique est instaurée selon une vision de performance, d'efficacité et d'efficience. De ce fait, Osbourne et Graebler (1992) incitent sur l'adoption d'une approche axée sur le client, et considèrent le citoyen comme un consommateur à satisfaire. Malgré les avantages offerts par la e-administration, la majorité des citoyens restent fidèles aux méthodes traditionnelles par le déplacement aux locaux de l'administration publique ou par le biais du téléphone (Fu, Fan, et Chao, 2006). 
Le choix du modèle de recherche doit intégrer les facteurs qui poussent le citoyen à l'usage des services publics en ligne. Dans ce sens, la problématique de l'adoption de l'administration électronique est proche de certaines problématiques traditionnelles de l'adoption de la technologie. Le modèle de recherche va donc s'appuyer sur des théories d'adoption des technologies, et mettra l'accent sur les déterminants de l'acceptation d'utilisation de la technologie pour l'accès aux services publics. Notre modèle de recherche va se baser sur la Théorie unifiée de l'acceptation et de l'utilisation de technologies (UTAUT). Cette théorie, comme nous l'avons expliqué cidessus, est un rassemblement de plusieurs modèles qui ont pour objectif d'expliquer les intentions d'utilisateurs des TICS et l'usage réel de ces dernières (Venkatech et al, 2003). Ainsi L'UTAUT a introduit quatre concepts fondamentaux qui influencent l'intention et l'usage :

- La performance attendue, l'effort attendu et l'influence sociale constituent des déterminants directs de l'intention qui explique à son tour l'usage effectif de l'individu des Tics.

- Les conditions facilitatrices influent directement sur le comportement d'utilisation sans passer par l'intention.

En outre, cette théorie intègre quatre variables modératrices qui sont: l'âge, le genre, l'expérience et le volontarisme. Au fur et à mesure que les travaux se développent dans cette discipline, des collaborations entre chercheurs se produisent ce qui a donné naissance à une UTAUT 2 (Venkatesh, Thong et $\mathrm{Xu}, 2012$ ), qui est une version révisée de l'UTAUT original en intégrant des facteurs émotionnels (le plaisir et l'hédonisme), et en ajoutant également le cout financier et les habitudes, les routines et les automatismes.

Sur la base de cette théorie avec ses construits qui sont bien expliqués ci-dessus à savoir, la performance, les conditions facilitatrices et les variables modératrices, notre modèle de recherche qui vise l'explication des déterminants de l'usage d'un système informatique: l'administration électronique, et qui va être testé à l'aide des données d'une enquête qui est en cours, est comme suit : 


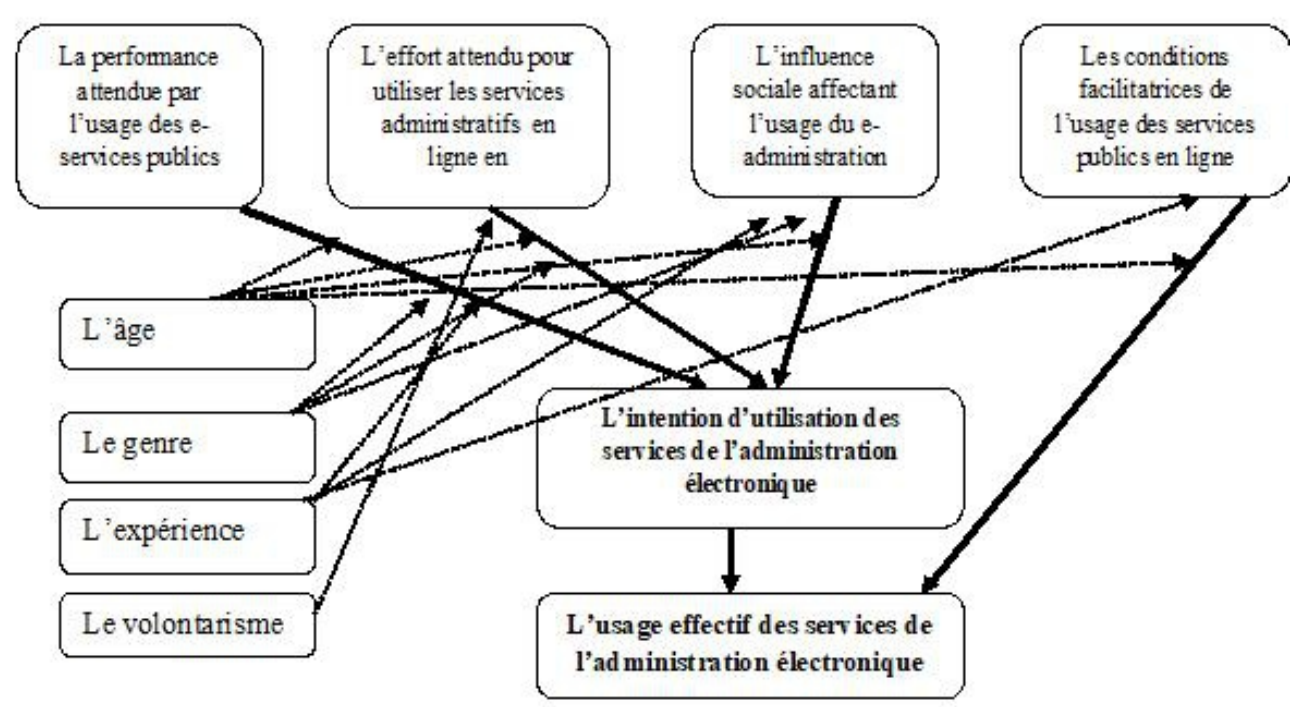

Le modèle de recherche sur l'acceptation d'usage des services de l'administration électronique adapté au modèle original de l'UTAUT (Venkatesh et al, 2003)

\section{Conclusion :}

Ce travail propose globalement une revue de littérature sur l'acceptation et l'adoption de l'utilisation des services électroniques par le citoyen. Dans ce sillage, nous avons encadré théoriquement la thématique de l'administration électronique, son adoption et son utilisation par le citoyen-usager. Ainsi, que la précision des facteurs qui influent sur cet usage.

En effet ce corpus théorique est composé de théories qui interviennent d'une manière homogène et complémentaire pour répondre à ce sujet d'actualité. En outre, nous ne pouvons pas parler de l'adoption et l'utilisation de la technologie sans parler de la théorie unifiée de l'acceptation et de l'utilisation de la technologie UTAUT. Cette théorie a connu plusieurs extensions au cours des années pour accompagner l'évolution des services en ligne, afin d'expliquer le comportement effectif de l'usage et le recours à ces services par le client-consommateur-usager. L'UTAUT est le résultat d'une fusion de plusieurs théories et modèles ayant pour objet aussi d'expliquer le comportement de l'utilisateur à l'égard du e-services, dont la théorie du comportement planifié, le modèle fondamental et ses trois extensions ( TAM 1, TAM 2, TAM 3), le modèle Motivationnel (MM), la théorie sociale cognitive, la théorie de la diffusion de l'innovation, la théorie d'influence sociale, des comportements interpersonnels et modèles d'utilisation des PC. Afin d'identifier les antécédents qui motivent l'usager à faire appel aux services électroniques, ces théories ont été axées sur des facteurs sociaux, cognitifs et instrumentaux. 
Cet article a pour but d'élaborer un modèle de recherche de l'acceptation et l'utilisation des e-services publics par le citoyen. En effet, Nous signalons que ce modèle représente que la base, et il ne s'agit que d'une étape préliminaire vers la compréhension de la problématique posée. Des recherches supplémentaires sont nécessaires pour modifier et intégrer d'autres variables au modèle de base. En outre, des travaux empiriques traitant le cas $\mathrm{du}$ Maroc seront prochainement établis, à travers une étude de collecte des données qui est en cours pour servir le test du modèle de recherche à l'aide d'une modélisation économétrique.

\section{References:}

1. Ajzen, I. (1991). The theory of planned behavior. Organizational behavior and human decision processes, 50(2), 179-211.

2. Ajzen, I. (1985). From intentions to actions: A theory of planned behavior. In Action control (pp. 11-39). Springer, Berlin, Heidelberg.

3. Ajzen, I., \& Fishbein, M. (1980). Understanding attitudes and predicting social behaviour.

4. Armitage, C. J., \& Conner, M. (1999). The theory of planned behaviour: Assessment of predictive validity and'perceived control. British journal of social psychology, 38(1), 35-54.

5. Attewell, P. (1992). Technology diffusion and organizational learning: The case of business computing. Organization science, 3(1), 1-19.

6. Bandura, A. (1977). Self-efficacy: toward a unifying theory of behavioral change. Psychological review, 84(2), 191.

7. Bandura, A. (1997). Self-Efficacy : the Exercise of Control . New York, Freeman, p. 422. p.422-425. p.427. p.189.

8. Bandura, A. (Ed.). (1995). Self-efficacy in changing societies. Cambridge university press.

9. Bandura, A. (1988). Organisational applications of social cognitive theory. Australian Journal of management, 13(2), 275-302.

10. Bandura, A. (1986). Social foundations of thought and action. Englewood Cliffs, NJ, 1986.

11. Banque Mondiale (2011). Définition of E-Government.

12. Coursey, D., \& Norris, D. F. (2008). Models of e-government: Are they correct? An empirical assessment. Public administration review, 68(3), 523-536.

13. Dada, D. (2006). The failure of E-government in developing countries: A literature review. The Electronic Journal of Information Systems in Developing Countries, 26(1), 1-10.

14. Davis, F. D., \& Venkatesh, V. (1996). A critical assessment of potential measurement biases in the technology acceptance model: 
three experiments. International Journal of Human-Computer Studies, 45(1), 19-45.

15. Davis, F. D., Bagozzi, R. P., \& Warshaw, P. R. (1992). Extrinsic and intrinsic motivation to use computers in the workplace 1. Journal of applied social psychology, 22(14), 1111-1132.

16. Davis, F. D. (1989). Perceived usefulness, perceived ease of use, and user acceptance of information technology. MIS quarterly, 319-340.

17. Fang, Z. (2002). E-government in digital era: concept, practice, and development. International journal of the Computer, the Internet and management, 10(2), 1-22.

18. Fu, J. R., Farn, C. K., \& Chao, W. P. (2006). Acceptance of electronic tax filing: A study of taxpayer intentions. Information \& Management, 43(1), 109-126.

19. Gefen, D. (2004). What makes an ERP implementation relationship worthwhile: Linking trust mechanisms and ERP usefulness. Journal of Management Information Systems, 21(1), 263-288.

20. Grönlund, Å. (Ed.). (2001). Electronic government: Design, applications and management: Design, applications and management. IGI Global.

21. Grönlund, A. (2005). State of the art in e-Gov research: Surveying conference publications. International Journal of Electronic Government Research, 1(4), 1-25.

22. Grönlund, Å., \& Horan, T. A. (2005). Introducing e-gov: history, definitions, and issues. Communications of the association for information systems, 15(1), 39.

23. Gupta, M. P., \& Jana, D. (2003). E-government evaluation: A framework and case study. Government information quarterly, 20(4), 365-387.

24. Stephen H, H., Norris, D. F., \& Fletcher, P. D. (2003). Electronic government at the local level: Progress to date and future issues. Public Performance \& Management Review, 26(4), 325-344.

25. Hiller, J. S., \& Bélanger, F. (2001). Privacy strategies for electronic government. E-government, 200, 162-198.

26. Karâa, M., \& Morana, J. (2011). Théorie de la diffusion de'innovation de Rogers et traçabilité: application au secteur de la datte tunisienne. Logistique \& Management, 19(1), 15-25.

27. Kefi, H. (2010). Mesures perceptuelles de l'usage des systèmes d'information: application de la théorie du comportement planifié. Humanisme et Entreprise, (2), 45-64.

28. Layne, K., \& Lee, J. (2001). Developing fully functional Egovernment: A four stage model. Government information quarterly, 18(2), 122-136. 
29. Lee, Y., Kozar, K. A., \& Larsen, K. R. (2003). The technology acceptance model: Past, present, and future. Communications of the Association for information systems, 12(1), 50.

30. Legris, P., Ingham, J., \& Collerette, P. (2003). Why do people use information technology? A critical review of the technology acceptance model. Information \& management, 40(3), 191-204.

31. Mitchell, S., \& Clark, D. (1999). Business adoption of information and communications technologies in the two-tier rural economy: some evidence from the South Midlands. Journal of rural studies, 15(4), 447-455.

32. Moon, M. J. (2002). The evolution of e-government among municipalities: rhetoric or reality? Public administration review, 62(4), 424-433.

33. Moon, M. J., \& Norris, D. F. (2005). Does managerial orientation matter? The adoption of reinventing government and e-government at the municipal level. Information Systems Journal, 15(1), 43-60.

34. Mossberger, K., Tolbert, C. J., \& Stansbury, M. (2003). Virtual inequality: Beyond the digital divide. Georgetown University Press.

35. OCDE (2003). The e-Government Imperative, OECD. Récupéré de http://www.oecdilibrary.org/governance/the-e-governmentimperative_9789264101197-en

36. Osborne, D., \& Gaebler, T. (1992). Reinventing government: How the entrepreneurial spirit is transforming government. Reading Mass. Adison Wesley Public Comp.

37. Peña-López, I. (2010). UN e-Government Survey 2010. Leveraging Egovernment at a Time of Financial and Economic Crisis.

38. Rogers, E. (2003). Diffusion of innovations. New York: Free Press, p. 473

39. Schmitz, J., \& Fulk, J. (1991). Organizational colleagues, media richness, and electronic mail: A test of the social influence model of technology use. Communication research, 18(4), 487-523.

40. Sheppard, B. H., Hartwick, J., \& Warshaw, P. R. (1988). The theory of reasoned action: A meta-analysis of past research with recommendations for modifications and future research. Journal of consumer research, 15(3), 325-343.

41. Stephen H, H., Norris, D. F., \& Fletcher, P. D. (2003). Electronic government at the local level: Progress to date and future issues. Public Performance \& Management Review, 26(4), 325-344.

42. St-Amant, G. (2016). E-Gouvernement: cadre d'évolution de l'administration électronique. Systèmes d'Information et Management, 10(1), 2. 
43. Sun, H., \& Zhang, P. (2006). The role of moderating factors in user technology acceptance. International journal of human-computer studies, 64(2), 53-78.

44. Taylor, S., \& Todd, P. A. (1995). Understanding information technology usage: A test of competing models. Information systems research, 6(2), 144-176.

45. Thomas, J. C., \& Streib, G. (2003). The new face of government: citizen-initiated contacts in the era of E-Government. Journal of public administration research and theory, 13(1), 83-102.

46. Thompson, R. L., Higgins, C. A., \& Howell, J. M. (1994). Influence of experience on personal computer utilization: testing a conceptual model. Journal of management information systems, 11(1), 167-187.

47. Triandis, H. C. (1971). Attitude and Attitude Change (Foundations of Social Psychology). New Jersey: John Wileys \& Sons Inc.

48. Un \& Aspa (2002). Benchmarking e-government: a global perspective. New York: United Nations Division of Public Economics and Public Administration and the American Society for Public Administration.

49. Venkatesh, V., Thong, J. Y., \& Xu, X. (2012). Consumer acceptance and use of information technology: extending the unified theory of acceptance and use of technology. MIS quarterly, 157-178.

50. Venkatesh, V., \& Bala, H. (2008). Technology acceptance model 3 and a research agenda on interventions. Decision sciences, 39(2), 273-315.

51. Venkatesh, V., Morris, M. G., Davis, G. B., \& Davis, F. D. (2003). User acceptance of information technology: Toward a unified view. MIS quarterly, 425-478.

52. Venkatesh, V., \& Davis, F. D. (2000). A theoretical extension of the technology acceptance model: Four longitudinal field studies. Management science, 46(2), 186-204.

53. Venkatesh, V. (2000). Determinants of perceived ease of use: Integrating control, intrinsic motivation, and emotion into the technology acceptance model. Information systems research, 11(4), 342-365.

54. West, D. M. (2000). Assessing e-government: The Internet, democracy and service delivery by state and federal governments.

55. Wood, R., \& Bandura, A. (1989). Social cognitive theory of organizational management. Academy of management Review, 14(3), 361-384. 